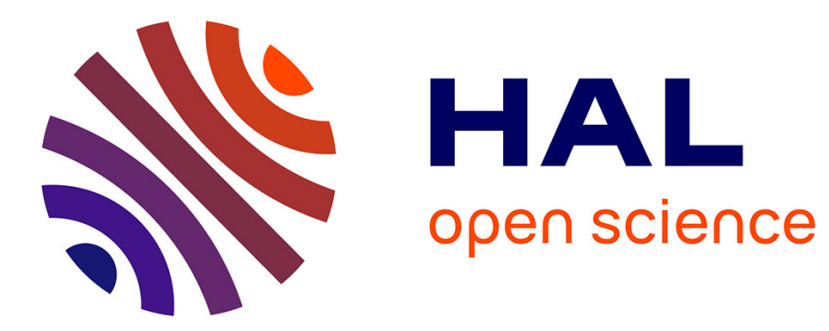

\title{
De l'inefficacité du réseau social: des liens sociaux non mobilisés chez les patients atteints de cancer
}

\author{
Adrien Defossez
}

\section{To cite this version:}

Adrien Defossez. De l'inefficacité du réseau social: des liens sociaux non mobilisés chez les patients atteints de cancer. Bulletin de Méthodologie Sociologique / Bulletin of Sociological Methodology, 2014, 121 (1), pp.44-58. 10.1177/0759106313509931 . halshs-01093409

\section{HAL Id: halshs-01093409 \\ https://shs.hal.science/halshs-01093409}

Submitted on 10 Dec 2014

HAL is a multi-disciplinary open access archive for the deposit and dissemination of scientific research documents, whether they are published or not. The documents may come from teaching and research institutions in France or abroad, or from public or private research centers.
L'archive ouverte pluridisciplinaire HAL, est destinée au dépôt et à la diffusion de documents scientifiques de niveau recherche, publiés ou non, émanant des établissements d'enseignement et de recherche français ou étrangers, des laboratoires publics ou privés. 


\section{De l'inefficacité du réseau social : des liens sociaux non mobilisés}

\section{Adrien Defossez}

LISST, Université de Toulouse

ISIS, Centre universitaire Champollion

\section{Résumé}

De nombreuses études sur les réseaux sociaux portent sur la question des ressources auxquelles un individu peut avoir accès à travers ses relations sociales. En s'intéressant aux réseaux qui ont «fonctionné », elles tentent alors de spécifier les facteurs ayant permis l'accès aux ressources. Mais peu de travaux se sont penchés sur les réseaux ayant échoué à fournir à l'individu le bien ou le service qu'il recherchait. Cet article se propose de discuter de ces réseaux «inefficaces » à travers le cas de patients atteints de cancer recherchant des informations relatives à leur pathologie. Les résultats indiquent que les causes de l'inefficacité du réseau sont à rechercher dans la non mobilisation des liens de la part des malades rencontrés. Un certain nombre de contraintes pèsent sur ces liens empêchant ainsi leur mobilisation.

Mots-clés : réseaux sociaux, inefficacité, mobilisation, ressources

\section{Introduction}

Une grande partie des études actuellement menées dans le champ des réseaux sociaux se concentre sur l'utilité que les acteurs peuvent retirer de leurs relations sociales. Cette conception instrumentale du réseau social est fortement liée à la notion de capital social, forgée dans les années 1980, d'abord chez Bourdieu, en complément des autres formes de 
capitaux (Bourdieu, 1980), puis chez Coleman qui étudie ses corrélations avec le capital humain (Coleman, 1988). Les travaux positionnés dans cette perspective s'intéressent aux ressources auxquelles un individu peut avoir accès à travers ses relations sociales ainsi qu'aux facteurs expliquant l'obtention de ces ressources (tels que la structure des réseaux et la nature des relations sociales). Une ressource peut être entendue comme tout bien ou service « dont la possession permet à l'individu de survivre ou de préserver ses acquis » (Lin, 1995) : un emploi, une aide pour déménager, un financement pour créer son entreprise, un moyen de locomotion, un soutien affectif, une information, etc.

Dans ces travaux, c'est bien l'efficacité du réseau social qui est analysée, c'est-à-dire la « capacité » de ce réseau à offrir à un individu la ressource qu'il cherche à obtenir. Les recherches se focalisent effectivement la plupart du temps sur les réseaux qui ont «bien fonctionné », en tentant, notamment, de caractériser la ou les relations personnelles ayant permis l'obtention de la ressource. L'étude de Granovetter portant sur le rôle du réseau social dans la recherche d'emploi illustre bien notre propos: après avoir indiqué que $56 \%$ des individus de son échantillon avaient obtenu leur emploi par leurs contacts personnels (et non par les démarches effectuées spontanément auprès des entreprises ou par le recours à des organismes institutionnels), Granovetter établit que ce sont les liens faibles, c'est-à-dire les contacts personnels les moins proches ${ }^{1}$, qui sont le plus efficaces du point de vue de la recherche d'emploi (Granovetter, 1974).

Cependant un réseau social peut également révéler son incapacité à fournir à un individu la ressource recherchée. S'il s'avère essentiel de mener une réflexion sur les avantages que les individus retirent de leurs relations sociales et leurs facteurs explicatifs, il est également intéressant de décrire les causes de l'inefficacité d'un réseau social. Ces deux

\footnotetext{
${ }^{1}$ Granovetter mesure la force d'un lien à travers le temps passé ensemble, l'intensité émotionnelle, la confiance mutuelle et les services réciproques (Granovetter, 1973).
} 
questionnements proches produisent des méthodologies d'enquête sensiblement différentes : quand le chercheur travaillant sur l'efficacité du réseau va s'attacher à recueillir des données sur des réseaux qui ont fonctionné («a obtenu la ressource »), le sociologue s'intéressant aux réseaux inefficaces va se concentrer sur les échecs et les difficultés. Les données travaillées ne sont donc pas exactement les mêmes et la question posée devient Pour quelles raisons le réseau social d'un individu ne lui permet pas d'obtenir la ressource qu'il recherche?

Plusieurs éléments d'explication ont déjà été avancés et peuvent être résumés par cette phrase tirée du manuel de Degenne et Forsé sur les réseaux sociaux: «Si un individu a besoin de l'aide de certains membres de son réseau pour atteindre un objectif, il faut bien sûr que ces derniers détiennent les ressources nécessaires, mais il faut surtout qu'ils soient prêts à lui accorder leur aide »(Degenne et Forsé, 2004, p123). Deux conditions paraissent, en effet, indispensables pour qu'un individu puisse obtenir de la part son réseau la ressource qu'il recherche. Il faut tout d'abord que ce que souhaite obtenir l'individu soit accessible à travers ses relations sociales, c'est-à-dire que la ressource soit présente dans son réseau et située à distance raisonnable ${ }^{2}$. Il est ensuite nécessaire que ses contacts soient coopératifs : dans le cas de liens sociaux négatifs, les relations sociales peuvent s'avérer être des freins à l'obtention des ressources. Ce peut être le cas de relations d'inimitié ou bien de relations de concurrence. Nous nous attacherons à décrire dans cet article un autre phénomène pouvant expliquer l'inefficacité d'un réseau social: les liens sociaux non mobilisés. Il est possible qu'un individu dispose d'un réseau social doté de la ressource recherchée, que ses contacts personnels soient coopératifs et que pour autant il n'arrive pas à obtenir cette ressource. Une personne peut en effet décider de ne pas mobiliser ses relations sociales : c'est le cas, par exemple, de doctorants ne recourant pas à leur directeur de thèse pour obtenir certaines

\footnotetext{
${ }^{2}$ Plus la distance, c'est-à-dire le nombre d'intermédiaires entre l'individu et la ressource, est élevée moins l'obtention de cette ressource parait envisageable. Granovetter note d'ailleurs dans son étude sur la recherche d'emploi que les chaines les plus courtes sont les plus efficaces (Granovetter, 1974).
} 
informations $^{3}$, d'individus ne sollicitant pas auprès de leurs connaissances de conseils en matière de sexualité (sur cet sujet voir Ferrand, 2007) ou bien encore d'un individu ne demandant pas aux membres de sa famille une aide financière dont il aurait pourtant besoin.

Afin de comprendre pourquoi certains liens sociaux, bien que pertinents, ne sont pas mobilisés, nous nous appuierons sur une étude menée auprès de patients. Au cours d'une enquête qui visait à déterminer par quels moyens des patients soignés en chimiothérapie ambulatoire obtenaient des informations concernant le cancer, le traitement et ses effets, il est apparu que de nombreuses questions que se posaient les malades restaient sans réponse. Nous avons alors voulu examiner plus avant cette situation et comprendre les causes de cette difficulté à obtenir les renseignements souhaités. La méthodologie de l'enquête axée sur ces situations d'échecs sera précisée dans une première partie. Par la suite, nous présenterons les causes de l'inefficacité du réseau social de ces patients en nous attachant à décrire les contraintes qui pèsent sur la mobilisation des différents liens sociaux pour enfin discuter du mécanisme de mobilisation des liens.

\section{A. Etude auprès de patients soignés en chimiothérapie ambulatoire}

\section{Traitement en ambulatoire et information des patients}

Une enquête qualitative a été menée, entre 2009 et 2010, dans un service hospitalier d'une ville moyenne du Sud de la France traitant des personnes atteintes de cancer. Celles-ci y sont soignées en chimiothérapie ambulatoire. Ce mode de traitement, privilégié en oncologie ${ }^{4}$, permet aux patients de venir à l'hôpital quelques heures dans une journée afin de recevoir

\footnotetext{
${ }^{3}$ Dans ce numéro du BMS, l'article de Bès et Chauvac discute précisément de cette situation.

${ }^{4}$ En France, seuls 9\% des patients recevant des soins de chimiothérapie anticancéreuse sont traités en hospitalisation complète (INCa, 2010, p13).
} 
leurs soins et rencontrer l'oncologue pour ensuite retourner à leur domicile. Selon les protocoles, les malades ne viennent à l'hôpital pour ces séances qu'une à quatre fois par mois. L'ambulatoire et les modes de prise en charge «hors-hôpital » de manière générale font l'objet d'une large promotion, tant au niveau institutionnel (FNEHAD, 2009 ; HAS, 2005) qu'académique (Caplan et al., 1999) : en permettant une autonomisation des patients et le maintien dans un environnement qui leur est familier, ceux-ci présenteraient des avantages en termes de qualité de vie par rapport à l'hospitalisation classique ${ }^{5}$.

Il paraissait alors pertinent, dans un contexte où le parcours de soins en cancérologie «manque encore trop souvent de continuité entre l'hôpital et le domicile » (voir l'Axe 4 du Plan cancer 2009-2013), de s'interroger sur la manière dont les patients hors-hôpital, qui ne sont dès lors plus continuellement en présence de personnels hospitaliers, s'informent sur leur pathologie et leur traitement. Les informations concernant la maladie, l'état de santé et le traitement revêtent pour le patient un caractère essentiel lorsqu'il s'agit d'expériences aussi lourdes que le cancer; elles sont, en effet, censées permettre au malade de faire face à l'incertitude médicale liée à cette pathologie (Ménoret, 2007, p49). Ainsi les questionnements concernant la compréhension de la maladie, sa gestion ainsi que la compréhension du traitement et des décisions médicales, c'est-à-dire l'ensemble des informations «pour connaitre » (Nabarette, 2002), font partie intégrante de l'expérience du malade. Une revue de littérature sur le sujet de l'information du malade dans le cadre du cancer indiquent que les études mettent largement en avant les bénéfices que les patients retirent de ces informations : elles permettraient au malade de participer davantage aux prises de décisions concernant sa pathologie, de réduire son anxiété et de mieux gérer sa maladie (Rutten et al., 2005). Mais

\footnotetext{
${ }^{5}$ Des auteurs nuancent toutefois ces discours avantageux en rappelant que le développement de ces modes de prise en charge procède davantage de contraintes budgétaires que de préoccupations sanitaires ou éthiques (Duke et Street, 2003) et indiquent que les gains en termes d'autonomie sont en réalité limités pour les patients horshôpital puisque ces modes de prise en charge reproduisent en partie «à l'extérieur » les contraintes habituellement rencontrées par les patients soignés en établissement hospitalier (Defossez et Ducournau, 2013).
} 
comment obtient-il ces informations si importantes ? Le patient s'appuie t-il sur son médecin traitant ? Réalise-t-il des recherches sur internet ? Contacte t-il le personnel de l'hôpital ? A-til recours à des associations de malades? A ses proches ? Etc. Telles sont les questions qui ont, au départ, guidé notre recherche auprès de ces patients atteints de cancer.

\section{Comment analyser les échecs?}

Lors des premiers entretiens réalisés, il est apparu que bon nombre de patients ont rencontré des difficultés pour obtenir certaines informations relatives à leur pathologie. Devant ce constat, il a été décidé de laisser dans le guide d'entretien une place à ces questionnements restés sans réponses. La méthodologie utilisée pour appréhender ces échecs se décompose en 3 étapes :

- Recenser les moyens mobilisables par le patient

Nous avons relevé différents moyens auxquels peuvent avoir recours les patients soignés en chimiothérapie ambulatoire pour obtenir une information de santé. Suivant la catégorisation effectuée par Lin, nous pouvons les regrouper selon deux types (Lin, 1995) : les moyens personnels et le réseau social. Le premier concerne les dispositifs directement mobilisables par les patients, c'est-à-dire sans faire appel à un contact personnel. Il s'agit ainsi des recherches sur internet, des émissions de télévision traitant de questions de santé, des revues et livres de médecine, des conférences sur la santé et des services d'aide aux patients (par exemple, cancer info service). Le second type fait référence aux opportunités offertes par le réseau social du malade. En effet, s'il recherche une information le patient a également la possibilité de s'appuyer sur ses relations sociales : il peut se rendre chez son médecin traitant ou un autre professionnel de santé (spécialistes, pharmaciens, médecins non-conventionnels, etc.), faire appel à ses proches, adhérer à une association de malades ou encore contacter l'oncologue de l'hôpital ou un autre personnel de ce service. 
La première étape consistait à déterminer, pour chaque patient, les moyens personnels ainsi que le réseau social «mobilisables ». Le terme mobilisable indique, parmi les solutions listées précédemment, celles dont disposent effectivement le patient interviewé et qu'il considère comme des moyens d'acquérir une information de santé. Il est possible qu'un patient ne possède pas de connexion internet (ou ne sache pas utiliser un moteur de recherche) et ne dispose pas, parmi son réseau social, de proches ayant des connaissances en matière de santé. Dans ce cas, ces deux possibilités ne seront pas admises comme des moyens mobilisables pour le patient considéré. De la même manière, si les émissions de télévision sur la santé peuvent être admises directement comme des moyens mobilisables (à partir du moment où le patient possède la télévision), il faut préciser que nombre de patients n'envisagent pas ce média comme une source d'information en matière de santé.

Afin d'obtenir les moyens personnels dont dispose le patient, nous le questionnons sur ses habitudes de vie et comportements associés à chacun de ces moyens : disposez-vous d'une connexion internet? Vous arrive-t-il d'utiliser des moteurs de recherche ? Etc. Pour ce qui est du réseau social, nous avons eu recours à un générateur de positions (Lin, 1999). Initialement, le générateur de positions consiste à présenter à un individu une liste de professions et à lui demander de citer des personnes qu'il connait exerçant ces métiers. Ce générateur permet d'obtenir la composition du réseau sur lequel l'individu peut s'appuyer pour acquérir des biens ou services spécifiques. Dans la présente étude, nous avons désigné des catégories professionnelles affiliées au domaine de la santé en demandant au patient s'il connaissait des personnes exerçant ces métiers ou s'il était en contact avec des associations de patients. Nous

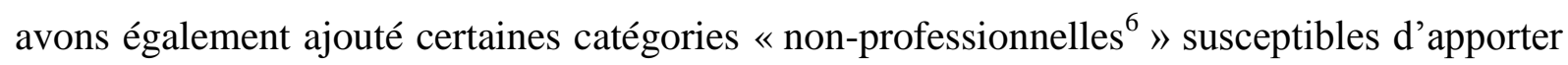
des informations médicales au patient : les proches disposant de certaines connaissances en

\footnotetext{
${ }^{6}$ «Non-professionnelles » dans le sens où le rôle principal de ce contact auprès du patient n'est pas d'être médecin ou pharmacien mais amis, parent ou simple connaissance. Ainsi dans cette catégorie nonprofessionnelle de contacts peuvent tout de même se retrouver des individus travaillant dans le milieu de la santé (par exemple une sœur infirmière).
} 
matière de santé et les alter ego ${ }^{7}$. Par ce procédé, nous recensons le réseau social mobilisable de chaque patient. Au final, nous obtenons l'ensemble des moyens mobilisables (moyens personnels et réseau social) pour chacun des patients rencontrés.

\section{- Rechercher les échecs}

Ensuite, en interrogeant les patients sur des thèmes ayant trait à l'expérience de la maladie, nous faisons ressortir certains questionnements apparus aux malades depuis le début de leur prise en charge. Il s'agit essentiellement d'interrogations portant sur la maladie elle-même (connaissance, évolution), le traitement (efficacité, médicaments, matériel) et ses effets indésirables, l'alimentation (nourriture préconisée, perte de poids) ainsi que sur la gestion de la maladie dans la vie quotidienne (activités envisageables, comportements et pratiques appropriés).

Nous demandons alors à chaque personne si elle a cherché à obtenir des réponses à ses questionnements et, le cas échéant, quel(s) moyen(s) parmi ceux dont elle dispose, elle a mobilisé pour y parvenir. Lorsque le patient nous indique qu'il n'a mobilisé ni ses moyens personnels ni son réseau social ou que le (ou les) moyen mobilisé s'est révélé inopérant, nous considérons que l'ensemble des moyens mobilisables se sont avérés inefficaces au regard de l'information recherchée («n'a pas obtenu la ressource »).

\section{- Comprendre les échecs}

Enfin, il est demandé au patient d'expliquer les raisons pour lesquelles il a choisi d'utiliser un moyen plutôt qu'un autre et en quoi celui-ci s'est révélé inefficace, ou bien pourquoi il n’a fait appel à aucune des possibilités qui s'offraient à lui. Ceci afin de comprendre les mécanismes qui sous-tendent les choix des patients dans l'utilisation des moyens d'information. Ainsi, quand un malade qui souhaitait obtenir une information concernant une douleur qu'il imputait

\footnotetext{
${ }^{7}$ Les Alter Ego représentent les fréquentations du patient qui sont actuellement traitées pour un cancer ou qui ont déjà été soignées pour cette maladie.
} 
à la chimiothérapie nous indique que sa recherche sur internet s'est avérée infructueuse, nous lui demandons dans un premier temps d'en préciser la raison. Ensuite nous le questionnons sur les autres moyens qu'il avait à sa disposition: pourquoi n'a-t-il pas contacté son pharmacien, son médecin traitant, sa belle-fille poursuivant des études de médecine ou pourquoi n'a-t-il pas appelé l'hôpital pour faire cette demande à l'oncologue qui le suit ? Le patient pourra, par exemple, indiquer qu'il pense que l'oncologue n'est pas disponible pour répondre à ce type de questions et que sa belle-fille ne connait pas encore grand chose au cancer et à ses traitements. Cette étape permet de faire ressortir les contraintes que les patients attribuent à chacun des moyens dont ils disposent, expliquant ainsi ce qui empêche leur mobilisation.

\section{Population interviewée}

Dans le cadre de cette étude, nous avons interviewé par entretiens semi-directifs 27 patients soignés en ambulatoire. Ces personnes ont été sollicitées pour participer à notre enquête le jour où elles se rendaient à l'hôpital, au moment où elles étaient en chambre de soin. Avant chaque entretien, nous nous sommes présentés à chacun des patients en tant que sociologue travaillant en collaboration avec l'hôpital sur une étude portant essentiellement sur la question de l'information du malade. Nous avons tenu systématiquement à souligner que nous ne faisions pas partie du personnel soignant et à préciser que nous sollicitions des entretiens dont le contenu resterait anonyme. Les entretiens furent enregistrés puis retranscrits in extenso.

Parmi les patients interrogés on trouve une proportion légèrement supérieure de femmes (15 femmes pour 12 hommes). Ceux-ci sont âgés de 23 à 82 ans avec un âge médian se situant à 62 ans. A l'exception d'un répondant, tous sont retraités ou en arrêt de travail. Si on retient le dernier emploi exercé, on peut noter une forte proportion (trois-quarts de l'échantillon) de personnes appartenant à des catégories sociales moyennes ou populaires (ouvrier du textile, 
serveur, magasinier, auxiliaire de vie, secrétaire...). Enfin, les expériences de la maladie sont hétérogènes puisqu'on trouve des patients qui viennent d'entrer dans le traitement quand d'autres ont une expérience du cancer de plusieurs années (16 d'entre eux ont débuté un premier traitement dans les 12 mois précédents et 11 ont reçu leur premier traitement il y a plus d'un an, dont 5 il y a plus de 3 ans) et que par ailleurs les types de cancer traités sont variables (sein, poumon, utérus, pancréas, maladie d'Hodgkin, leucémie, etc.).

\section{B. L’inefficacité du réseau social des patients}

\section{Les moyens personnels des patients}

Si cet article se propose de discuter des situations où le réseau des patients s'est avéré inefficace, il nous faut néanmoins présenter les résultats concernant les moyens directement mobilisables par les malades. En effet, quand un patient n'obtient pas l'information souhaitée c'est bien parce que l'ensemble des moyens mobilisables s'est avéré inefficace, le réseau social comme les moyens personnels.

Parmi les 27 patients interviewés, 14 disposent d'une connexion Internet à leur domicile, leur offrant ainsi la possibilité de rechercher des informations concernant leur pathologie. Mais ces patients, qui se sont surtout connectés «au début », «pour voir » disent avoir fait l'expérience d'une source d'information qui leur a été inutile. A l'exception d'un individu qui, étant donné son métier d'infirmier, dit arriver à trier et comprendre les informations trouvées sur Internet, les patients rencontrés admettent avoir eu des difficultés à localiser les informations qu'ils recherchaient, à les comprendre et ont exprimé leur manque de confiance dans cette source 
d'information. Ils craignent d'une part l'inexactitude des renseignements trouvés et d'autre part que ceux-ci ne se rapportent pas à leur situation personnelle ${ }^{8}$.

Le problème de la personnalisation des informations recueillies se retrouve également en ce qui concerne d'autres moyens personnels mobilisables par les patients comme les émissions de télévision et les revues portant sur le domaine de la santé. Si certaines enquêtes indiquent que ces sources d'information, relativement peu mobilisées par nos patients ${ }^{9}$, sont considérées par les individus comme plus fiables qu'internet (Dumitru et al., 2007), il apparait tout aussi difficile pour les patients de notre échantillon d'y sélectionner les informations pertinentes, c'est-à-dire relatives à leur cas personnel. Quant aux dispositifs d'information institutionnels, mis en place, par exemple par l'INCa ou la Ligue contre le cancer (par exemple Cancer info), ils restent pour la plupart inconnus des patients rencontrés.

$\mathrm{Au}$ final, lorsqu'un patient présente une situation d'échec vis-à-vis d'une information recherchée et que nous le questionnons sur les moyens personnels dont il disposait, il s'avère que ces sources d'information sont considérées comme peu fiables et insuffisamment personnalisées pour répondre à des questionnements sur son cancer et son traitement.

\section{Quand le réseau social se révèle inopérant}

Du côté du réseau social, la question de la fiabilité des sources informations est également un élément pouvant expliquer certains échecs. Celle-ci ne se situe plus au niveau de la confiance accordée à la source d'information, comme ce pouvait être le cas avec Internet, mais davantage au niveau de la compétence que les patients attribuent à leurs différentes relations sociales concernant le cancer. En effet, si les patients ont des contacts avec leur médecin

\footnotetext{
${ }^{8}$ Sur la mise en cause de la fiabilité des informations disponibles sur internet voir également les études de Friedman et al., 2006 et Hargrave et al., 2003.

${ }^{9}$ Par exemple, seul un quart des patients rencontrés envisagent les émissions de télévision sur la santé comme une source d'information concernant leur pathologie.
} 
traitant, leur pharmacien ou des spécialistes extérieurs à l'hôpital, ils ne recherchent pas leur avis de professionnel médical pour cette pathologie. Le seul avis qui les intéresse réellement est celui de l'oncologue de l'hôpital et, dans une moindre mesure, celui des infirmiers du service. Beaucoup de patients ne s'appuient donc pas sur ces liens sociaux que l'on aurait pu s'attendre à voir mobilisés. Même s'ils parlent de leur maladie à leur médecin traitant, les discussions engagées restent relativement superficielles comme en témoigne cet extrait d'entretien : « [Concernant le médecin traitant] Je considère que c'est pas son truc [la cancérologie]. Je lui apprends un peu ce que je fais ici, la dernière fois que je l'ai vu je lui ai donné les noms des 4 médicaments que j'allais prendre, il était très content parce qu'il ne les savait pas, il les a notés. A la limite c'est plutôt moi qui le renseignerais, enfin si on peut dire, tout en restant raisonnable. Je considère que c'est pas sa partie, donc je lui demande pas ».

Le patient peut discuter de sa situation avec son médecin traitant pour «me rassurer, me soulager » comme l'exprime une autre patiente, mais il ne considère pas celui-ci comme un interlocuteur compétent concernant le cancer. Lors d'un entretien, une patiente nous répondra également dans ce sens après une question de relance portant sur l'aptitude du pharmacien à la renseigner sur les médicaments susceptibles de réduire les effets indésirables de la chimiothérapie (notamment les vomissements) : «Oui, le pharmacien peut savoir certaines choses, c'est sûr, mais je le juge pas compétent comme... [l'oncologue] ».

En ce qui concerne les proches, les facteurs explicatifs de leur non mobilisation sont doubles. Les deux tiers des malades rencontrés ont des proches qui, de par leurs expériences professionnelles ou personnelles, seraient susceptibles de leur fournir des renseignements concernant leur maladie (membres de la famille exerçant le métier d'infirmier, amis médecins ou bien proches ayant déjà vécu l'expérience du cancer). Lorsqu'il est question de cette possibilité, les patients invoquent, comme pour le médecin traitant ou le pharmacien, la logique de compétence, ce qui confirme les résultats d'une enquête portant sur la fiabilité que 
les individus accordent à cette source d'information (Le Queau et al., 2001). Mais ils indiquent également leur volonté de ne pas entrer dans une relation soignant-soigné avec ces membres de leur réseau, comme le souligne cette patiente : «Oui, j’ai des amis médecins avec qui je travaillais, avec qui je suis restée en bons termes.[...] J'aime pas justement les embêter aussi dans la mesure où c'est des amis au-delà du côté médical, je veux pas avoir l'air de les embêter sur un plan médical. On parle de livres, de films, de festival d'Avignon où on va ensemble ou de choses comme ça mais surtout pas... [du cancer] ».

La crainte de cette patiente de franchir la frontière entre lien d'amitié et lien médical est probablement liée aux pratiques de ce corps de métier. Comme l'avait déjà remarqué Héran dans une étude sur la sociabilité, certaines professions (notamment les professions de la santé et du travail social) évitent en effet de mélanger pratiques amicales et pratiques professionnelles (Héran, 1987, p47). Un autre patient, directeur d'établissement de santé à la retraite, confirme cette idée :

«Les toubibs que je connais bien c'est des amis, on se fend la gueule ensemble, on se fait des bonnes bouffes, ça c'est important. Chacun à sa place. »

Ainsi, les patients peuvent éviter de faire appel à leurs proches ayant certaines connaissances en matière de santé par crainte de faire basculer la relation amicale ou familiale dans une relation gouvernée par des préoccupations médicales. Il ne faut pas pour autant penser que cette crainte serait uniquement présente du côté du malade : dans certaines situations c'est peut-être en effet davantage les proches qui font comprendre au malade qu'ils ne souhaitent pas basculer dans une relation soignant-soigné. Si les proches peuvent constituer une ressource importante sur le plan émotionnel et pour gérer le quotidien de la maladie (Bury, 1982 ; Carricaburu et Pierret, 1995 ; Smith et Midanik, 1980), cette proximité expliquerait également en partie leur non mobilisation pour l'obtention d'autres types de ressources, comme des informations relatives à leur pathologie dans notre étude. 
Enfin quand l'oncologue, seul interlocuteur jugé compétent pour des questions liées au cancer (avec, à un degré moindre, les infirmiers de l'hôpital), n'est pas mobilisé c'est parce que les patients ne souhaitent pas importuner ce contact qu'ils jugent déjà très occupé. La représentation des personnels hospitaliers débordés, largement partagée au sein de la population, semble se renforcer une fois vécue l'expérience hospitalière. Ainsi, un patient indique se restreindre dans ses appels téléphoniques afin de se démarquer de certains comportements qu'il juge excessifs : "J'ai toujours peur de déranger. Bon, c'est vrai que quand c'est pour déplacer un rendez-vous ou un truc comme ça je peux difficilement faire autrement mais quand je vois ce qui se passe au niveau des coups de téléphone, il y en a qui appellent pour n'importe quoi. [...] Je fais gaffe quand même, justement je suis prudent à cause de ça parce que je ne veux pas déranger. [...]». Imaginant ce que peuvent dire les soignants, il rajoute «Ne nous gonflez plus, on a autre chose à faire que de répondre au téléphone ».

Une patiente exprime également cette crainte de déranger le personnel, alors qu'elle ressent des crampes dans tout le corps depuis la dernière séance de chimiothérapie : «Je n'ai pas osé appeler l'hôpital parce qu'ils ont tellement de boulot, ils sont tellement pris », puis elle ajoute : «On a mal mais on n'ose pas le dire, sinon je vais passer pour douillette ».

Ce sentiment d'avoir affaire à un personnel débordé, associé à la crainte de renvoyer une image de «mauvais patient»(Coser, 1962; Lorber, 1975), embêtant le service pour des questions qui pourraient être perçues comme sans importance, expliquent certains échecs relevés concernant la recherche d'information. L'extrait d'entretien suivant dévoile cette volonté de ne pas renvoyer l'image d'un patient dérangeant: «Ca c'est des questions que je me pose moi et que je n'ose pas demander au cancérologue de peur d'être ridicule, qu'on me dise attendez vous gambergez ou ceci ou cela, ça c'est moi. Ils doivent tellement en entendre ces pauvres médecins qu'ils doivent en avoir marre. [...] Je n'aime pas les surcharger avec 
des questions qu'elles doivent entendre à longueur de journées, j'ai l'impression d'être un peu gnian-gnian et de vouloir qu'on s'occupe que de moi ».

Tout se passe comme si les patients n'usaient pas de ce lien social en dehors des séances de chimiothérapie à l'hôpital afin de ne pas risquer de voir la relation se détériorer. Ceci d'autant plus dans un contexte où le malade est suivi par le personnel hospitalier pendant des mois, voire des années. Cette contrainte de l'image renvoyée a déjà pu être relevée dans des situations de co-présence médecin-patient au sein de l'établissement hospitalier (Fainzang, 2006), mais la situation «hors hôpital» semble renforcer cette crainte, limitant ainsi les possibilités de mobilisation de ce lien social.

Les échecs dans l'obtention d'information de santé qui ont pu être relevés chez les patients rencontrés s'expliquent donc par la combinaison de l'inefficacité des moyens personnels et du réseau social des malades. Le problème de la fiabilité des sources d'information, tant au niveau de la confiance (pour les moyens personnels, comme Internet) que de la compétence des contacts pour des questionnements liés au cancer (médecin traitant, pharmacien, proches), s'ajoute au souci de maintenir les relations avec les proches sur un plan affectif et de ne pas dégrader la relation avec le personnel hospitalier. Au final, il est possible d'identifier trois profils de patients vis-à-vis de la recherche d'information de santé.

- Le patient autonome

Ces patients actifs, se réattribuent leur maladie et «jonglent» avec les différentes ressources à leur disposition. Le patient autonome recherche des informations à travers les différents moyens dont il dispose : il utilise fréquemment internet pour des questions relatives à sa maladie et dispose de certaines compétences indispensables pour trier et comprendre les informations qu'il y trouve. Il se construit un véritable réseau social de santé qu'il mobilise pour obtenir un conseil, confirmer un diagnostic ou encore interpréter des résultats d'examens. Au final il n'hésite pas à mettre en concurrence la parole des différents acteurs qui 
l'entourent dans son expérience de malade et à confronter leurs dires aux informations qu'il obtient par le biais des médias, tel qu'internet. Nous n'avons identifié qu'un individu correspondant à cette catégorie parmi les malades interrogés : il s'agit du patient relativement jeune (47 ans) exerçant le métier d'infirmier. Cette position lui permet d'une part de disposer de compétences en matière de santé nécessaires à une utilisation efficace des informations qu'il obtient et d'autre part de ne pas être inhibé par le système hospitalier qu'il connait bien. Outre le profil de professionnel de santé, on pourrait également retrouver dans cette catégorie des patients issus de milieux favorisés et ayant déjà une bonne connaissance de l'expérience hospitalière. Il semblerait qu'en cancérologie, les patients autonomes soient plus rares que dans d'autres maladies puisque comme l'indique Ménoret, "la cancérologie transfère peu de compétences aux malades qu'elle traite » $(2007, \mathrm{p} 81)$, contrairement au diabète par exemple.

\section{- Le patient désarmé}

Ce profil de patient correspond au malade impliqué dans sa pathologie et son traitement mais n'ayant pas les compétences nécessaires à une gestion autonome de la maladie. Le patient désarmé recherche dès le diagnostic (parfois avant) des informations concernant son cancer et son traitement mais s'aperçoit rapidement qu'il ne dispose pas des connaissances pour pouvoir trier et utiliser les informations se rapportant à sa situation. Son réseau social de santé est relativement limité (souvent un alter Ego ou un proche travaillant dans le milieu de la santé en plus du médecin traitant et du personnel hospitalier) et il n'ose pas le mobiliser pour des questionnements précis sur le cancer. Les proches sont davantage présents pour fournir du soutien émotionnel et gérer le quotidien. Quant au personnel hospitalier, le malade ne souhaite pas risquer de détériorer la relation qu'il a noué avec celui-ci en «dérangeant » le service oncologie pour des questions qui pourraient être jugées comme sans importance. Ainsi, si les ressources que le patient recherche sont bien présentes dans son réseau social, il ne peut les 
obtenir puisqu'il ne mobilise pas ses liens sociaux. Ce profil concerne deux tiers des patients rencontrés.

- Le patient isolé

Ce dernier profil renvoie au patient se déchargeant de toutes responsabilités et se laissant porter par l'institution hospitalière. Son réseau social de santé se limite au personnel hospitalier et à son médecin traitant (quand il n'a pas rompu avec ce dernier, comme c'est le cas pour deux de ces patients) ; en ce sens, il est possible de parler d'un malade relativement isolé. Il n'envisage à aucun moment de rechercher des informations par lui-même à travers d'autres interlocuteurs, comme les proches ou des professionnels de santé extérieurs à l'hôpital. Par ailleurs, les médias, comme internet ou les revues de santé, ne sont pas perçus comme des sources d'informations mobilisables concernant le cancer. Au final, seul l'oncologue (et dans une moindre mesure les infirmiers) est jugé compétent. Mais celui-ci n'est pas pour autant mobilisé : le malade se contente d'attendre les moments opportuns de questionnements, comme lorsque l'oncologue passe dans la chambre au moment des séances de chimiothérapie. En ce qui concerne la recherche d'informations relatives au cancer, le patient est donc totalement dépendant de l'institution hospitalière. Un peu moins d'un tiers des patients interrogés correspondent à ce profil.

\section{Discussion et conclusion}

\section{Dotation du réseau en ressources}

Comme précisé dans la première partie, les patients interrogés sont principalement issus de catégories sociales moyennes, voire populaires. Et c'est probablement la présence majoritaire de ces statuts sociaux peu élevés dans la population étudiée qui a permis de déceler autant de 
situations d'échec relatives à la recherche d'information de santé. En effet, de nombreuses études menées sur le capital social indiquent que le statut social d'un individu explique en partie les possibilités que lui offre son réseau social en matière d'obtention de ressources (voir par exemple, Lai et al., 1998 ; Lin, 1999). Ainsi, en réalisant cette enquête auprès d'une population plus aisée, nous aurions vraisemblablement rencontré plus de patients connaissant personnellement des « experts » sur le sujet du cancer ou bien des patients davantage investis dans des associations de malades. Il en est de même du côté des moyens personnels mobilisables : une population privilégiée serait sans doute plus à même de trier et comprendre les informations recueillies sur Internet et de déterminer celles qui se rapportent à son cas personnel ou bien aurait la possibilité de le demander aux contacts appropriés de son réseau social. Au final, nous pouvons dire que les possibilités offertes à notre population en termes d'accès aux informations de santé étaient moindres, ce qui a surement influencé statistiquement les résultats concernant l'obtention de ces ressources. Il apparait logique que lorsque son réseau social est peu doté de la ressource recherchée, l'individu a moins de chances de l'obtenir.

Pour autant, chacun des patients rencontrés disposait parmi ses relations sociales d'au minimum un contact direct (l'oncologue) ayant la possibilité de fournir l'information recherchée. Ainsi, si le niveau social d'un patient peut donner une indication quant à la dotation de son réseau en ressources, il ne saurait constituer le facteur déterminant de l'obtention de celles-ci. L'effet quantitatif ne permet aucunement d'expliquer ce qui se joue dans les relations interpersonnelles au niveau de la transmission des ressources. La question reste alors entière : qu'est ce qui empêche un individu d'obtenir une ressource présente dans son réseau ? Notre enquête a montré que les facteurs explicatifs étaient à rechercher dans le mécanisme de mobilisation des liens sociaux. 
Cette étude sur l'inefficacité du réseau social des malades nous a permis de relever différentes raisons pour lesquelles les patients n'avaient pas eu recours à leurs relations sociales. Nous savons qu'il s'agit tout d'abord du problème de la compétence que les patients attribuent à certains de leurs contacts sur des questions relatives au cancer (médecin traitant, pharmacien, proches). Considérant que ces personnes ne disposent pas de l'information qu'ils recherchent, ils ne vont pas mobiliser ces liens. Il n'est pas nécessaire d'expliquer plus avant ce phénomène qui traduit simplement la représentation qu'un individu a des ressources possédées par les membres de son réseau.

Le $2^{\text {ème }}$ type de non mobilisation est beaucoup plus intéressant : le lien avec l'oncologue, seul informateur jugé compétent, n'est pas activé pour ne pas risquer de dégrader la relation. Ainsi des individus peuvent ne pas mobiliser un lien dont ils savent pourtant qu'il leur permettrait d'obtenir la ressource recherchée. Si les patients craignent de détériorer la relation avec le personnel soignant, c'est qu'ils ont l'impression qu'appeler l'hôpital pour poser une question sur un médicament par exemple, ou une douleur à une jambe, serait jugé comme abusif de la part de l'oncologue et du personnel hospitalier: «cela ne se fait pas ». Les patients ont effectivement intégré la règle selon laquelle il ne faut pas appeler le service oncologie pour poser des questions «sans importance ». La contrainte ne pas déranger émane de règles plus ou moins tacites qui structurent la relation du patient avec l'oncologue. De façon générale, ces règles structurent, au moins en partie, les relations que le patient entretient avec chacun des membres de son réseau. Dans une étude sur les confidents, Alexis Ferrand remarque que $61 \%$ des individus masculins appartenant à la catégorie sociale cadre et professions intellectuelles supérieures ne citent aucun membre de leur réseau, en dehors de leur conjoint, comme confident en matière de sexualité (Ferrand, 2007). Comme le note l'auteur, ce fait ne provient pas d'une absence de liens mobilisables mais d'un tabou. Du fait de cet interdit, ces individus ne peuvent pas mobiliser leurs liens sociaux pour cette ressource. Ségolène Petite note 
également la présence d'interdits dans la mobilisation des liens à propos d'une enquête sur l'entraide ; elle définit ainsi les contraintes relationnelles issues des normes sociales : «Un premier mode de régulation repose sur les modèles de conduite associés aux rôles incarnés par les partenaires impliqués dans une relation. Ces modèles facilitent l'ajustement des manières d'agir des uns vis-à-vis des autres en précisant ce qui est idéalement interdit, obligé ou possible, voire souhaitable, avec ses différents partenaires »(Petite, 2005, p43).

De manière générale, nous pouvons recenser trois types de contraintes pouvant peser sur la mobilisation des relations sociales. Pour cela, nous reprenons les différentes dimensions du triangle des régulations développé par Alexis Ferrand (Ferrand, 2007). Ce dernier indique qu'une relation sociale est gouvernée par des références normatives et les modalités de contrôle qui leur sont associées, celles-ci sont au nombre de trois et sont simultanément prises en compte par les acteurs (Ferrand, 2007, p107). Il s'agit des normes générales, des normes propres à une clique du réseau (par exemple, les relations de voisinage) et enfin des règles émanant de la relation dyadique (c'est à dire de la relation interpersonnelle). Si l'auteur définit ces modes de régulation pour classer les systèmes relationnels et étudier leur dynamique, cette idée peut également permettre de comprendre le mécanisme de mobilisation des liens sociaux et ainsi mettre en lumière un des facteurs de l'inefficacité des réseaux sociaux.

Les trois types de contraintes qui pèsent sur les liens sociaux expriment ce que nous pouvons appeler «l'activabilité des liens sociaux », c'est-à-dire la possibilité pour un individu de mobiliser une relation pertinente afin d'obtenir une ressource. Lorsque les contraintes attachées à une relation sont trop fortes au regard de la ressource recherchée, l'individu ne mobilisera pas ce lien. A travers le cas de patients qui n'ont pas contacté l'oncologue pour obtenir certaines informations, nous avons pu ainsi observer les contraintes émanant des normes sociales. La contrainte ne pas déranger le personnel soignant, largement partagée au 
sein de la population de malades et relayée sous d'autres formes ${ }^{10}$ par l'institution hospitalière, constitue, pour ces patients, une règle que l'on ne peut enfreindre sans risquer de voir la relation avec l'équipe soignante se dégrader.

Un autre exemple tiré de notre enquête portait sur une patiente, infirmière à la retraite, qui indiquait ne pas contacter ses amis médecins pour des questions liées au cancer et préférait que les préoccupations médicales n'interfèrent pas dans leur relation amicale. Cette nonmobilisation renvoie, quant à elle, au deuxième mode de régulation des relations défini par Ferrand, c'est-à-dire les normes issues du réseau de relations. Cette règle, particulière au groupe d'anciens collègues qui désire ne pas mélanger pratiques amicales et pratiques professionnelles, spécifie la conduite à tenir uniquement envers ces contacts. Pour ce qui est des contraintes propres aux relations dyadiques ( $3^{\text {ème }}$ mode de régulation), il pourrait s'agir d'un patient ne demandant pas à un ami ayant eu un cancer des informations pratiques sur la pathologie (par exemple concernant l'alimentation ou les effets secondaires) parce qu'il sait que ce dernier ne souhaite pas lui parler de son expérience. Ici, ce serait bien la connaissance intime du contact, de ses attentes et l'histoire spécifique de leur relation qui expliquerait la non-activation.

Il parait ainsi envisageable de déceler la présence de ces type de contraintes (normes sociales, normes du réseau, règles dyadiques) à travers une étude systématique de l'inefficacité des réseaux sociaux. Ceci pour les situations de recherche d'informations en santé comme nous l'avons fait ici, mais également pour ce qui est de l'accès à un emploi, à un aide financière, un conseil ou de manière générale pour n'importe quelles situations où un individu cherche à obtenir une ressource. En étudiant les liens positifs, c'est-à-dire ceux qui ont permis l'obtention de la ressource, il n'est pas possible de mettre à jour ces contraintes. Il faut, en

\footnotetext{
${ }^{10}$ Ainsi, il est demandé aux patients de contacter en priorité leur médecin traitant en cas de problèmes et non l'hôpital. Cela est d'ailleurs précisé dans le livret d'information remis à chaque patient en début de traitement.
} 
effet, passer par les échecs et les impossibilités exprimés par les individus pour déceler les contraintes qui pèsent sur les liens sociaux et ainsi déterminer les raisons de l'inefficacité d'un réseau social.

\section{Références}

- Bourdieu P. (1980) Le capital social. Notes provisoires, Actes de la recherche en sciences sociales, 31, 2-3.

- Bury M. (1982) Chronic illness as a biographical disruption, Sociology of health and illness, 4, 2, 167-182.

- Caplan, G.A., Ward, J.A., Brennan, N.J., Coconis, J., Board, N., and Brown, A. (1999) Hospital in the home: a randomised controlled trial, The medical journal of Australia, $170,4,156-160$

- Carricaburu, D., and Pierret, J. (1995) From biographical disruption to biographical reinforcement: the case of HIV-positive men, Sociology of health and illness, 17, 1, $65-88$

- Coleman J. (1988) Social capital in creation of human capital, American journal of Sociology, 94, 95-120.

- Coser, R.L. (1962) Life in the Ward. East Leasing: Michigan State University Press

- Defossez, A. et Ducournau, P., 2013, Patients receiving ambulatory care: a problematic autonomy situation, Health Sociology Review, Accepté pour publication.

- Degenne A. et Forsé M., 2004, Les réseaux sociaux, une analyse structurale, Armand Colin.

- Duke, M., and Street, A. (2003) The impetus for the development of Hospital In The Home (HITH) programs: a literature review, Contemporary Nurse, 14, 3, 227-239 
- Dumitru, R.C., Bürkle T., Potapov T., Lausen B., Wiese B., Prokosch H.U. (2007) Use and perception of internet for health related purposes in Germany: results of a national survey, International Journal of Public Health, 52, 275-285.

- Fainzang S. (2006) La relation médecins-malades : information et mensonge, Paris, PUF, Coll. Ethnologies.

- Ferrand A. (2007) Confidents. Une analyse structurale de réseaux sociaux, Paris, L'Harmattan, Coll. Logiques sociales.

- FNEHAD (2009) Fédération Nationale des Etablissements d'Hospitalisation A Domicile, Livre Blanc des systèmes d'information en hospitalisation à domicile, Institutional Report, 143p.

- Friedman, D.B., Hoffman-Goetz, L., and Arocha, J.F. (2006) Health literacy and the World Wide Web: Comparing the readability of leading incident cancers on the Internet, Medical Informatics and the Internet in Medicine, 31, 1, 67-87

- Granovetter M. (1973) The strength of weak ties, American journal of sociology, 78, $6,1360-1380$.

- Granovetter M. (1974) Getting a Job: A Study of Contacts and Careers. Cambridge, MA: Harvard University Press.

- Hargrave, D.R., Hargrave, U.A., and Bouffet, E. (2006) Quality of health information on the internet in pediatric neuro-oncology, Neuro-oncology, 8, 2, 175-182.

- HAS (2005) Analyse comparée de la chimiothérapie anticancéreuse administrée à l'hôpital ou prise ne charge à domicile: aspects économiques et organisationnels, Rapport HAS, 92p.

- Héran, F. (1987) Comment les français voisinent, Economie et statistique, 195, 43-59.

- INCa (2010) Situation de la chimiothérapie des cancers en 2010, Coll. Rapports \& synthèses, ouvrage collectif édité par l'INCa, Boulogne-Billancourt, 38p. 
- $\quad$ Lai, G., Lin, N. and Leung S-Y (1998) Network resources, contact resources, and status attainment, Social networks, 20, 159-178.

- Le Queau, P., Olm, C. et Simon, M-O (2001) L'information en matière de santé, Rapport pour le Credoc, 113p.

- Lin N. (1995) Les ressources sociales : une théorie du capital social, Revue française de sociologie, 36, 4, 685-704.

- Lin N. (1999) Social Networks and Status attainment, Annual review of sociology, 25, 467-487.

- Lorber J. (1975) Good patients and problem patients: conformity and deviance in a general hospital, journal of health and social behavior, 16, 2, 213-225

- Ménoret M. (2007b) Les temps du cancer, Le bord de l'eau.

- Nabarette H. (2002) L'internet médical et la consommation d'information par les patients, Réseaux, 114, 249-286

- Petite S. (2005) Les règles de l'entraide. Sociologie d'une pratique sociale, Presses universitaires de Rennes, Coll. Le sens social.

- $\quad$ Rutten L.J., Arora N.K., Bakos A.D., Aziz N. \& Rowland J. (2005) Information needs and sources of information among cancer patients : a systematic review of research (1980-2003), Patient education and counseling, 57, 3, 250-261.

- Smith, R.T., and Midanik, L. (1980) The effects of social resources on recovery and perceived sense of control among the disabled, Sociology of Health and Illness, 2, 1, $48-63$ 